\title{
A Pilot Study- Neoadjuvant Chemotherapy with Gemcitabine and S1 in Patients with Resectable and Borderline Resectable Pancreatic Cancer
}

\author{
Akira Matsushita $^{1 *}$, Yoshiharu Nakamura1 ${ }^{1}$, Hiroki Sumiyoshi ${ }^{1}$, Takayuki Aimoto $^{1}$, Tadashi Yokoyama $^{2}$, and Eiji Uchida ${ }^{1}$ \\ ${ }^{1}$ Departments of Gastrointestinal and Hepatobiliary-Pancreatic Surgery, Nippon Medical School, Tokyo, Japan \\ ${ }^{2}$ Department of Surgery, Nippon Medical School, Tama Nagayama Hospital, Nippon Medical School, Tokyo, Japan
}

\begin{abstract}
Introduction: Combination chemotherapy with gemcitabine and S-1 (GS) in metastatic advanced pancreatic cancer patients is superior to gemcitabine alone in response rate and progression free survival. We investigated this combination chemotherapy as neoadjuvant therapy for resectable and borderline resectable pancreatic cancer

Methods: Eleven patients with resectable or borderline resectable pancreatic cancer were administered to neoadjuvant chemotherapy with GS (NeoGS) from June 2011 to March 2013 at Nippon Medical School, and shortterm outcome was evaluated.

Results: The median age was 69.1 years. According to NCCN criteria, 6 patients were resectable diseases and 5 were borderline resectable diseases. All patients received Neo GS with a median cycle of 3.5 (range: 2-11). No serious adverse events including death or life-threatening complications happened. Grade 3 or 4 chemotherapyrelated toxicities included neutropenia $(81.8 \%)$, anemia $(18.2 \%)$, thrombocytopenia $(18.2 \%)$, and febrile neutropenia $(9.1 \%)$. Other non-hematological toxicities with grade 1 or 2 were anorexia $(36.4 \%)$, constipation (36\%), nausea $(27.3 \%)$, diarrhea $(18.2 \%)$, dysgeusia $(9.1 \%)$, and stomatitis $(9.1 \%)$. Radiologically, partial response was documented in 3 patients $(27.3 \%)$, and the remaining 8 patients $(72.7 \%)$ had stable disease. All patients underwent pancreatic resection with lymphadenectomy. An R0 resection was achieved in 10 of 11 (90.9\%), and negative nodal involvement (N0) was found in 6 (54.5\%). Pathologically, all specimens showed at least Evans grade I, while eight of eleven $(72.7 \%)$ had Evans grade Ila. There was no mortality and severe morbidity including clinically relevant pancreatic fistula. All patients received adjuvant chemotherapy with either gemcitabine or S1.
\end{abstract}

Conclusion: This pilot study suggests NeoGS is feasible in patients with resectable and borderline resectable pancreatic cancer and may be associated with a high R0 resection rate and a low lymph node metastasis rate, suggesting that further phase 2 and 3 trials are warranted.

Keywords: Resectable and borderline resectable pancreatic cancer; Neoadjuvant chemotherapy; Gemcitabine; S-1; Gemcitabine+S-1

\section{Introduction}

Pancreatic cancer is a $5^{\text {th }}$ leading cause of cancer death and causes approximately 28,800 deaths per year in Japan [1]. Pancreatic cancer has an extremely poor prognosis with a 5 -year overall survival of less than $5 \%$ [2]. Pancreatic resection remains the only hope for long-term survival, but unfortunately only $15-20 \%$ of patients with pancreatic cancer are capable of undergoing pancreatic resection [3]. To improve the survival of patients with pancreatic cancer, increasing rate of resection with negative surgical margins (R0 resection) and decreasing rate of metastatic Lymph Nodes (LN) are important [3]. Recently, randomized control trials demonstrated adjuvant chemotherapy with gemcitabine after curative resection of pancreatic cancer significantly prolonged disease free survival compared with surgery alone but did not significantly prolonged overall survival $[4,5]$. The major problem of adjuvant chemotherapy is that a large proportion of patients cannot receive any treatment due to preoperative morbidity after pancreatic resection [6].

On the contrary, neoadjuvant treatment can be applied to almost all patients since it is independent of the perioperative morbidity. In fact, several randomized phase 2 trials in resectable pancreatic cancer demonstrated that neoadjuvant chemotherapy with gemcitabine and cisplatin was associated with a higher resection rate and a favorable survival rate $[7,8]$. S-1 is a fourth generation oral fluoropyrimidine, which combines tegafur with two 5-flurouracil modulators, gimeracil, which is a 200 -fold more potent inhibitor of dihydropyrimidine dehydrogenase than uracil and potassium oxonate, which can reduce the diarrhea caused by Tegafur at a molar ratio of 1 to 0.4 to 1 (Tegafur: gimeracil: potassium oxonate) [9]. A phase 2 trial of S-1 for advanced metastatic pancreatic cancer have shown a response rate of $37.5 \%$ and the median time to progression and the overall survival time were 3.7 months and 9.2 months [10]. Moreover, multicenter randomized phase 2 trials of a combination of gemcitabine and S-1 (GS) for advanced metastatic pancreatic cancer showed the significant superiority of GS in response rate and progression free survival, but not in overall survival when compared to gemcitabine alone $[11,12]$. GS as neoadjuvant therapy in patients with resectable or borderline resectable pancreatic cancer may be effective with respect to progression and survival.

However, a trial of neoadjuvant chemotherapy with gemcitabine and S-1 (NeoGS) for resectable and borderline resectable pancreatic cancer has not been demonstrated. Therefore, we conducted a pilot

*Corresponding author: Akira Matsushita, Department of Gastrointestinal and Hepato-biliary-Pancreatic Surgery, Nippon Medical School, 1-1-5 Sendagi, Bunkyo-ku, Tokyo, 113-8603 Japan, Tel: +81.3.3822.213; Fax: +81.3.5685.6075; E-mail: akira-matsushita@nms.ac.jp

Received May 06, 2013; Accepted July 02, 2013; Published July 12, 2013

Citation: Matsushita A, Nakamura Y, Sumiyoshi H, Aimoto T, Yokoyama T, et al. (2013) A Pilot Study- Neoadjuvant Chemotherapy with Gemcitabine and S1 in Patients with Resectable and Borderline Resectable Pancreatic Cancer. J Carcinogene Mutagene S9: 006. doi:10.4172/2157-2518.S9-006

Copyright: (c) 2013 Matsushita A, et al. This is an open-access article distributed under the terms of the Creative Commons Attribution License, which permits unrestricted use, distribution, and reproduction in any medium, provided the original author and source are credited. 
Citation: Matsushita A, Nakamura Y, Sumiyoshi H, Aimoto T, Yokoyama T, et al. (2013) A Pilot Study- Neoadjuvant Chemotherapy with Gemcitabine and S1 in Patients with Resectable and Borderline Resectable Pancreatic Cancer. J Carcinogene Mutagene S9: 006. doi:10.4172/21572518.S9-006

Page 2 of 5

study of NeoGS for patients with resectable and borderline resectable pancreatic cancer

\section{Patients and Methods}

\section{Patients}

Between June 2011 and March 2013, 11 patients who were diagnosed as histologically or cytologically proven resectable or borderline resectable pancreatic adenocarcinoma with Eastern Cooperative Oncology Group (ECOG) performance status of 0 or 1 and adequate organ function at Nippon Medical School were included. All patients were planned to perform neoadjuvant chemotherapy with GS followed by surgery as anti-cancer treatment. A written informed consent was obtained from all patients.

\section{Staging and resectability criteria}

Contrast-enhanced (CE) CT scan of chest and abdomen ruled out distant metastases. Positron-emmision tomography (PET) CT was also used for detecting unexpected distant metastases, if available. Local resectability was assessed by relationship between tumor extent and major visceral vessels, including Superior Mesenteric Artery (SMA), Celiac Artery (CA), Common Hepatic Artery (CHA), and Portal Vein (PV) or superior mesenteric vein (SMV), based on the CECT. We applied the National Comprehensive Cancer Network (NCCN) Guidelines criteria for definition of resectable or borderline resectable pancreatic cancer $[13,14]$. These criteria are summarized in Table 1.

\section{Neoadjuvant and adjuvant chemotherapy}

Neoadjuvant chemotherapy consists of $1,000 \mathrm{mg} / \mathrm{m}^{2}$ gemcitabine given as an intravenous infusion over $30 \mathrm{~min}$ on dayl and day8, and $30 \mathrm{mg} / \mathrm{m}^{2} \mathrm{~S} 1$ given orally twice daily on days 1 to 14 , every 3 weeks. Patients with resectable pancreatic cancer received 2 cycles and patients with borderline resectable pancreatic cancer received more than 2 cycles.

After pancreatic resection, patients received adjuvant chemotherapy with $1000 \mathrm{mg} / \mathrm{m}^{2}$ gemcitabine on dayl, day 8 , and day 15 every 4 weeks or $40 \mathrm{mg} / \mathrm{m}^{2} \mathrm{~S} 1$ twice daily on days 1 to 28 , every 6 weeks for 6 months.

\section{Surgery}

Surgery was performed within 10-30 days after the last neoadjuvant chemotherapy. All patients underwent pancreatic resection with lymphadenectomy. In cases with suspected tumor infiltration into the PV and/or SMV, concomitant resection of those veins with reconstruction was performed. The operation was defined as an R0 resection if there was no microscopic tumor found at the margin and as an $\mathrm{R} 1$ resection if a margin was microscopically positive.

\section{Response and toxicity assessments and perioperative complications}

Tumor responses were measured by CECT or magnetic resonance imaging scans by comparing between the baseline and after neoadjuvant chemotherapy according to the Response Evaluation Criteria in Solid Tumors (RECIST) version 1.0. Adverse events during chemotherapy were graded by the "Common Terminology Criteria for Adverse Events" version 4.0. The International Study Group on Pancreatic Fistula Definition defined pancreatic fistula [15].

\section{Pathologic response}

To assess the pathologic effects of neoadjuvant chemotherapy, the grading system reported by Evans et al. was used [16]. Briefly, the Evans grading system consists of scale from I to IV based on the degree of cytological change and tumor destruction as follows: grade I refers to characteristic cytologic changes of malignant cells are present, but little (below 10\%) or no tumor cell destruction is evident; grade IIa, destruction of 10 to $50 \%$ of tumor cells; grade IIb, destruction of 51 to $90 \%$ of tumor cells; grade III, few (below $10 \%$ ) viable-appearing tumor cells present; grade IV, no viable tumor cells are present.

\section{Results}

\section{Patient's characteristics}

From June 2011 and March 2013, 11 patients (9 males and 2 females) were diagnosed as resectable or borderline resectable pancreatic cancer, and received neoadjuvant chemotherapy with gemcitabine and S1. The baseline characteristics of these patients are summarized in Table 2 . According to the NCCN criteria, 6 patients had resectable diseases and 5 patients had borderline resectable diseases. Of those who had borderline disease, three patients had abutment or encasement of the SMV, one had abutment of the SMA, and one had both encasement of the SMV and abutment of the SMV (Figures 1a and 1b).

\begin{tabular}{|l|l|}
\hline Category & Criteria \\
\hline Resectable & $\begin{array}{l}\text { No distant metastases } \\
\text { No evidence of SMV and portal vein abutment, distortion, tumor } \\
\text { thrombus or venous encasement Clear fat planes around the celiac } \\
\text { axis, hepatic artery, and SMA. }\end{array}$ \\
\hline $\begin{array}{l}\text { No distant metastases } \\
\text { Venous involvement of the SMV/portal vein demonstrating tumor } \\
\text { abutment with or without impingement and narrowing of the lumen, } \\
\text { encasement of the SMV/portal vein but without encasement of } \\
\text { the nearby arteries, or short segment venous occlusion resulting } \\
\text { from either tumor thrombus or encasement but with suitable vessel } \\
\text { proximal and distal to the area of vessel involvement, allowing for } \\
\text { safe resection and reconstruction. } \\
\text { Gastroduodenal artery encasement up to the hepatic artery with } \\
\text { resectabler short segment encasement or direct abutment of the hepatic } \\
\text { either shy } \\
\text { arteryithout extension to the celiac axis. } \\
\text { Tumor abutment of the SMA not to exceed } 180 \text { degrees of the } \\
\text { circumference of the vessel wall }\end{array}$ \\
\hline
\end{tabular}

Abbreviations: SMA- Superior Mesenteric Artery; SMV- Superior Mesenteric Vein Table 1: Criteria used for determining local resectability for pancreatic cancer

\begin{tabular}{|c|c|c|}
\hline Characteristic & Number & $\%$ \\
\hline $\begin{array}{c}\text { Age at diagnosis, yr } \\
\text { Median(range) }\end{array}$ & $69.1(59 \sim 78)$ & \\
\hline Sex & & \\
\hline Male & 9 & 81.8 \\
Female & 2 & 18.2 \\
\hline ECOG Performance status & 10 & 90.9 \\
\hline 0 & 1 & 9.1 \\
\hline 1 & 10 & 90.9 \\
\hline Site of tumor & 1 & 9.1 \\
\hline Head & 6 & 27.5 \\
\hline Tail & 3 & 9.1 \\
\hline $\begin{array}{c}\text { Resectability } \\
\text { Resectable }\end{array}$ & 1 & 9.1 \\
\hline Borderline resectable \\
SMV encased (short segment)
\end{tabular}

Abbreviations: SMV- Superior Mesenteric Vein; SMA- Superior Mesenteric Artery Table 2: Patient characteristics, $n=11$. 
Citation: Matsushita A, Nakamura Y, Sumiyoshi H, Aimoto T, Yokoyama T, et al. (2013) A Pilot Study- Neoadjuvant Chemotherapy with Gemcitabine and S1 in Patients with Resectable and Borderline Resectable Pancreatic Cancer. J Carcinogene Mutagene S9: 006. doi:10.4172/21572518.S9-006

\section{Toxicity and adverse events}

All 11 patients received adequate neoadjuvant therapy with gemcitabine and $\mathrm{S} 1$ with a median cycle of 3.5 (range: $2-11$ ). No serious
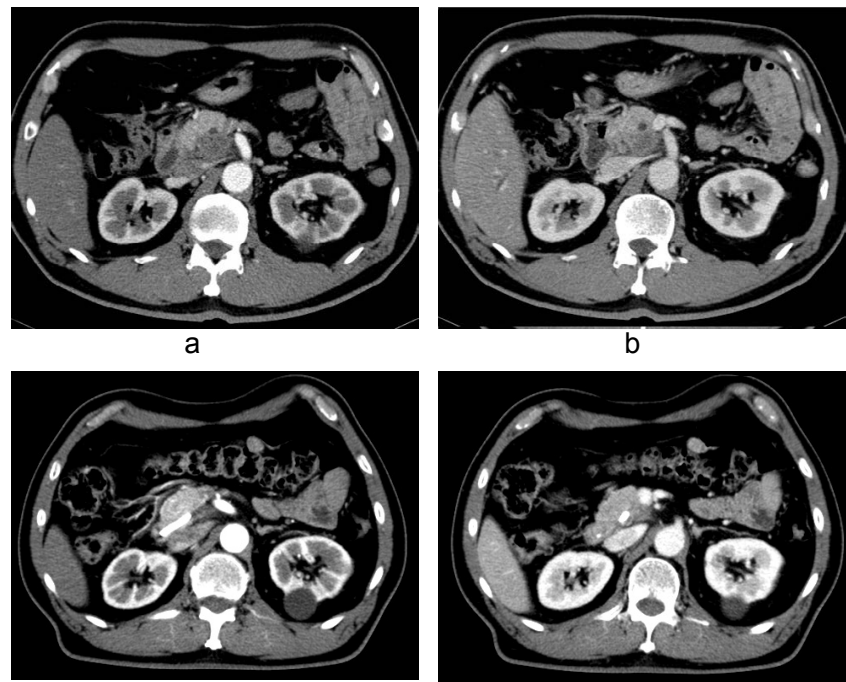

C

d

Figure 1: A 64-year-old man was diagnosed with pancreatic head cancer. The SMA abutment and the SMV involvement were detected in CECT (a, b). He was diagnosed as borderline resectable according to NCCN criteria. After 3 cycles of neoadjuvant chemotherapy with gemcitabine and S1, The tumor at pancreas head had regressed, and SMA abutment were improved but SMV encasement still existed in CECT(c, d) (partial response). He underwent laparoscopic subtotal stomach preserving Pancreaticoduodenectomy with lymphadenectomy and the SMV resection. The operation was R0 resection and the pathologic stage was T1N0 (case 9).

\begin{tabular}{|c|c|c|}
\hline & Grade $\mathbf{3}$ or $\mathbf{4}$ & $\%$ \\
\hline Neutropenia & 9 & 81.8 \\
\hline Anemia & 2 & 18.2 \\
\hline Thrombocytopenia & 2 & 18.2 \\
\hline Febrile neutropenia & 1 & 9.1 \\
\hline
\end{tabular}

Other non-hematological toxicities with grade 1 or 2 were anorexia $(36.4 \%)$, constipation $(36 \%)$, nausea $(27.3 \%)$, diarrhea $(18.2 \%)$, dysgeusia $(9.1 \%)$, and stomatitis $(9.1 \%)$.

Table 3: Grade3 or 4 adverse events associated with gemcitabine and S1 neoadjuvant therapy. adverse events including death or life-threatening complications happened. As shown in Table 3, most common hematological toxicities with grade 3 or 4 were neutropenia $(81.8 \%)$, anemia (18.2\%), thrombocytopenia (18.2\%), and febrile neutropenia (9.1\%). Other non-hematological toxicities with grade 1 or 2 were anorexia $(36.4 \%)$, constipation $(36.4 \%)$, nausea $(27.3 \%)$, diarrhea (18.2\%), dysgeusia (9.1\%), and stomatitis (9.1\%).

\section{Radiologic tumor response}

None of the patients showed an increase in tumor size during NeoGS on CECT. Partial response was documented in 3 patients (27.3\%, Figures $1 \mathrm{c}$ and $1 \mathrm{~d})$, and the remaining 8 patients had stable disease (72.7\%) (Table 4).

\section{Surgical outcome}

After radiologic reevaluation using CECT, all patients were deemed resectable or borderline resectable after neoadjuvant therapy. All patients underwent pancreatic resection with lymphadenectomy. The $\mathrm{R} 0$ resection rate was $90.9 \%$ (10 patients) and $\mathrm{R} 1$ resection rate was $9.1 \%$ (1 patient) (Table 4 ). None of patients had perioperative death and severe postoperative morbidities including pancreatic fistula graded B or C.

\section{Pathologic response}

Histopathologic assessment of the resected specimen in the 11 patients who received NeoGS is summarized in Table 4. Most of the pancreatic tumors located in the head of the pancreas $(\mathrm{N}=10 ; 90.0 \%)$. Most of the patients had T3N1M0 tumors $(\mathrm{N}=5 ; 45.5 \%)$. Negative lymph node metastasis was found in 6 patients (54.5\%). With respect to pathologic response, minimal response was present in 8 patients (grade IIa: $\mathrm{N}=8 ; 72.7 \%$ ).

\section{Discussion}

Recently, results of a large randomized phase 3 trial of GS and gemcitabine alone in unresectable pancreatic cancer patients, known as the GEST trial, were reported. This large-scale $(\mathrm{N}>600)$ trial demonstrated significant superiority of GS in response rate and progression free survival but not in overall survival when compared to gemcitabine alone [17].

The current pilot study evaluated the feasibility and initial therapeutic effect of NeoGS in resectable and borderline resectable pancreatic cancer patients. The patients who received NeoGS had

\begin{tabular}{|c|c|c|c|c|c|c|c|c|c|c|c|c|}
\hline Patient & $\begin{array}{l}\text { Clinical } \\
\text { T stage }\end{array}$ & $\begin{array}{l}\text { Clinical } \\
\mathrm{N} \text { stage }\end{array}$ & Site & Criteria & Factor & $\begin{array}{l}\text { No. of } \\
\text { cycle }\end{array}$ & $\begin{array}{l}\text { RECIST } \\
\text { response }\end{array}$ & Surgery & $\begin{array}{l}\text { Pathologic } \\
\text { T stage }\end{array}$ & $\begin{array}{l}\text { Pathologic } \\
\text { N stage }\end{array}$ & $\begin{array}{l}\text { Pathologic } \\
\text { response }\end{array}$ & $\begin{array}{l}\text { Surgical } \\
\text { margin }\end{array}$ \\
\hline 1 & 4 & 1 & $\mathrm{H}$ & BR & SMA & 4 & SD & SSPPD & 3 & 1 & Ila & Ro \\
\hline 2 & 3 & 0 & $\mathrm{H}$ & $\mathrm{BR}$ & SMV & 4 & PR & SSPPD/SMVR & 3 & 0 & Ila & Ro \\
\hline 3 & 1 & 0 & $\mathrm{H}$ & $\mathrm{R}$ & - & 2 & SD & SSPPD & 1 & 0 & I & Ro \\
\hline 4 & 3 & 0 & $\mathrm{H}$ & $\mathrm{R}$ & - & 2 & SD & SSPPD & 3 & 1 & I & R0 \\
\hline 5 & 3 & 0 & $\mathrm{H}$ & $\mathrm{BR}$ & SMV & 2 & SD & SSPPD/SMVR & 3 & 0 & 1 & R0 \\
\hline 6 & 3 & 1 & $\mathrm{~T}$ & $\mathrm{R}$ & - & 11 & PR & DP & 3 & 1 & Ila & Ro \\
\hline 7 & 3 & 0 & $\mathrm{H}$ & $\mathrm{R}$ & - & 2 & SD & Lap-PD & 3 & 1 & Ila & Ro \\
\hline 8 & 3 & 0 & $\mathrm{H}$ & $\mathrm{R}$ & - & 2 & SD & Lap-SSPPD & 3 & 0 & Ila & $\mathrm{R} 1$ \\
\hline 9 & 4 & 0 & $\mathrm{H}$ & $\mathrm{BR}$ & SMVISMA & 3 & PR & Lap-SSPPD/SMVR & 1 & 0 & Ila & Ro \\
\hline 10 & 3 & 0 & $\mathrm{H}$ & $\mathrm{BR}$ & SMV & 4 & SD & Lap-SSPPD/SMVR & 3 & 1 & Ila & Ro \\
\hline 11 & 3 & 0 & $\mathrm{H}$ & $\mathrm{R}$ & - & 2 & SD & Lap-SSPPD & 3 & 0 & Ila & R0 \\
\hline
\end{tabular}

Abbreviations: RECIST- Response Evaluation Criteria In Solid Tumors; H- Head of Pancreas; T- Tail of Pancreas; SMA- Superior Mesenteric Artery; SMV- Superio Mesenteric Vein; SD- Stable Disease; PR- Partial Response; SSPPD- Subtotal Stomach Preserving Pancreaticoduodenectomy; SMVR- Superior Mesenteric Vein Resection; DP- Distal Pancreatectomy; Lap-PD- Laparoscopic Pancreaticoduodenectomy; Lap-SSPPD- Laparoscopic Subtotal Stomach Preserving Pancreaticoduodenectomy 
Citation: Matsushita A, Nakamura Y, Sumiyoshi H, Aimoto T, Yokoyama T, et al. (2013) A Pilot Study- Neoadjuvant Chemotherapy with Gemcitabine and S1 in Patients with Resectable and Borderline Resectable Pancreatic Cancer. J Carcinogene Mutagene S9: 006. doi:10.4172/21572518.S9-006

\begin{tabular}{|c|c|c|c|c|c|c|c|c|c|}
\hline Year & Auther & Patients & $\begin{array}{c}\text { Borderline } \\
\text { N (\%) }\end{array}$ & Chemotherapy & $\begin{array}{l}\text { Radiation } \\
\text { (Gy) }\end{array}$ & $\begin{array}{c}\text { Resection rate } \\
\mathrm{N}(\%)\end{array}$ & $\begin{array}{c}\mathrm{R} 0 \text { resection } \\
\mathrm{N}(\%)\end{array}$ & $\begin{array}{c}\text { Negative LN } \\
\text { metastasis: } \mathrm{N}(\%)\end{array}$ & $\begin{array}{l}\text { Median overall } \\
\text { survival(M) }\end{array}$ \\
\hline 2007 & Palmer [7] & $\begin{array}{l}24 \\
26\end{array}$ & $\begin{array}{l}- \\
-\end{array}$ & $\begin{array}{c}\text { Gem } \\
\text { Gem, Cis }\end{array}$ & $\begin{array}{l}- \\
-\end{array}$ & $\begin{array}{c}8(37.5 \%) \\
18(69.2 \%)\end{array}$ & $\begin{array}{c}6(75 \%) \\
12(46.2 \%)\end{array}$ & $\begin{array}{l}2(25 \%) \\
7(44 \%)\end{array}$ & $\begin{array}{c}9.9 \\
15.6\end{array}$ \\
\hline 2008 & Heinrich [8] & 28 & - & Gem, Cis & - & $25(89.3 \%)$ & $20(80 \%)$ & - & 19.1 \\
\hline 2008 & Varadhachary [18] & 90 & - & Gem, Cis & 30 & $52(58 \%)$ & $50(96.2 \%)$ & $20(38.5 \%)$ & 31 \\
\hline 2009 & Turrini [19] & 102 & - & $5 \mathrm{FU}, \mathrm{Cis}$ & 45 & $62(60.8 \%)$ & $57(91.9 \%)$ & $47(75.8 \%)$ & 23 \\
\hline 2011 & Patel [20] & 17 & $17(100 \%)$ & Gem, TXT, Cap & 50 (5FU) & $9(64 \%)$ & $8(88.9 \%)$ & - & 15.6 \\
\hline 2011 & Sahora [21] & 33 & $15(45.5 \%)$ & Gem, Ox & - & $13(39 \%)$ & $9(69.2 \%)$ & $4(30.8 \%)$ & 22 \\
\hline 2012 & Lee [22] & 43 & $18(41.9 \%)$ & Gem, Cap & - & $17(39.5 \%)$ & $14(82.4 \%)$ & $7(41.2 \%)$ & 23.1 \\
\hline \multirow[t]{2}{*}{2012} & Hosein [23] & 18 & $4(23 \%)$ & FOLFIRINOX & - & $9(50 \%)$ & $8(88.9 \%)$ & - & - \\
\hline & This study & 11 & $5(45.5 \%)$ & Gem, S1 & - & $11(100 \%)$ & $10(90.9 \%)$ & $6(54.5 \%)$ & - \\
\hline
\end{tabular}

Abbreviations: LN- Lymph Node; Gem- Gemcitabine; Cis- Cisplatin; TXT- Docetaxel; Cap- Capecitabine; Ox- Oxaliplatin; M- Month

Table 5: Neoadjuvant chemotherapy in resectable and borderline resectable pancreatic cancer.

neither death nor life-threatening serious adverse events, but most of the patients had neutropenia or other hematological toxicities with grade 3 or 4 . NeoGS in patients with resectable and borderline resectable pancreatic cancer resulted in an $\mathrm{R} 0$ resection rate of $90.9 \%$ with a negative lymph node metastasis rate of $54.5 \%$.

Some neoadjuvant chemotherapy regimens for resectable or borderline resectable pancreatic cancer are reported. As summarized in Table 5, those regimens were gemcitabine alone, gemcitabine plus cisplatin, 5FU plus cisplatin, gemcitabine plus oxaliplatin, gemcitabine, docetaxel plus capecitabine, gemcitabine plus capecitabine, and FOLFILINOX [18-23]. The FOLFILINOX regimen consists of 5-fluorouracil, leucovorin, irinotecan and oxaliplatin. The randomized phase 3 trial demonstrated FOLFILINOX in patients with metastatic pancreatic cancer was significantly superior to gemcitabine alone in response rate, progression free survival and overall survival [24]. Most of all regimens were based on combination chemotherapy with gemcitabine. Although the patient characteristics of those studies were different in resectability criteria, in combination chemotherapies, R0 resection rates were reported with a range from $66.7 \%$ to $96.2 \%$, and negative lymph node metastasis rates were from $30.8 \%$ to $75.8 \%$. In this study, R0 resection rate and negative lymph node rate were similar to those of other combination chemotherapies. Although Neo GS showed a high $\mathrm{R} 0$ resection rate and a low lymph node metastasis rate in resectable and borderline resectable pancreatic cancer, the findings of pathological response was limited.

In conclusion, this pilot study suggests that NeoGS is feasible in patients with resectable and borderline resectable pancreatic cancer and may be associated with a high $\mathrm{R} 0$ resection rate and a low lymph node metastasis rate. Randomized phase 2 and 3 trials of NeoGS in patients with resectable or borderline resectable pancreatic cancer are warranted for making sure if NeoGS would prolong survival for patients with resectable and borderline resectable pancreatic cancer.

\section{Acknowledgement}

The authors declare no competing interests and no financial interests.

\section{References}

1. Ministry of Health, Labour and Welfare (2011) Trends in deaths and death rates (per 100,000 populations) from malignant neoplasms by sex and site: Japan in 2011.

2. Siegel R, Naishadham D, Jemal A (2013) Cancer statistics, 2013. CA Cancer J Clin 63: 11-30.

3. Sohn TA, Yeo CJ, Cameron JL, Koniaris L, Kaushal S, et al. (2000) Resected adenocarcinoma of the pancreas-616 patients: results, outcomes, and prognostic indicators. J Gastrointest Surg 4: 567-579.
4. Oettle H, Post S, Neuhaus P, Gellert K, Langrehr J, et al. (2007) Adjuvant chemotherapy with gemcitabine vs observation in patients undergoing curativeintent resection of pancreatic cancer: a randomized controlled trial. JAMA 297 267-277.

5. Ueno H, Kosuge T, Matsuyama Y, Yamamoto J, Nakao A, et al. (2009) A randomised phase III trial comparing gemcitabine with surgery-only in patients with resected pancreatic cancer: Japanese Study Group of Adjuvant Therapy for Pancreatic Cancer. Br J Cancer 101: 908-915.

6. Russ AJ, Weber SM, Rettammel RJ, Mahvi DM, Rikkers LF, et al. (2010) Impact of selection bias on the utilization of adjuvant therapy for pancreas adenocarcinoma. Ann Surg Oncol 17: 371-376.

7. Palmer DH, Stocken DD, Hewitt H, Markham CE, Hassan AB, et al. (2007) A randomized phase 2 trial of neoadjuvant chemotherapy in resectable pancreatic cancer: gemcitabine alone versus gemcitabine combined with cisplatin. Ann Surg Oncol 14: 2088-2096

8. Heinrich S, Pestalozzi BC, Schäfer M, Weber A, Bauerfeind P, et al. (2008) Prospective phase II trial of neoadjuvant chemotherapy with gemcitabine and cisplatin for resectable adenocarcinoma of the pancreatic head. J Clin Oncol 26: 2526-2531.

9. Shirasaka T (2009) Development history and concept of an oral anticancer agent S-1 (TS-1): its clinical usefulness and future vistas. Jpn J Clin Oncol 39 2-15.

10. Okusaka T, Funakoshi A, Furuse J, Boku N, Yamao K, et al. (2008) A late phase II study of S-1 for metastatic pancreatic cancer. Cancer Chemothe Pharmacol 61: 615-621.

11. Nakai Y, Isayama H, Sasaki T, Sasahira N, Tsujino T, et al. (2012) A multicentre randomised phase II trial of gemcitabine alone vs gemcitabine and S-1 combination therapy in advanced pancreatic cancer: GEMSAP study. $\mathrm{Br}$ Cancer 106: 1934-1939.

12. Ozaka M, Matsumura $Y$, Ishii H, Omuro $Y$, Itoi T, et al. (2012) Randomized phase II study of gemcitabine and S-1 combination versus gemcitabine alone in the treatment of unresectable advanced pancreatic cancer (Japan Clinical Cancer Research Organization PC-01 study). Cancer Chemother Pharmacol 69: $1197-1204$

13. Callery MP, Chang KJ, Fishman EK, Talamonti MS, William Traverso L, et al. (2009) Pretreatment assessment of resectable and borderline resectable pancreatic cancer: expert consensus statement. Ann Surg Oncol 16: 1727 1733

14. National Comprehensive Cancer Network (NCCN) Clinical Practice Guidelines Pancreatic Adenocarcinoma. [Accessed March 28,2013]

15. Bassi C, Dervenis C, Butturini G, Fingerhut A, Yeo C, et al. (2005) Postoperative pancreatic fistula: an international study group (ISGPF) definition. Surgery 138 8-13.

16. Evans DB, Rich TA, Byrd DR, Cleary KR, Connelly JH, et al. (1992) Preoperative chemoradiation and pancreaticoduodenectomy for adenocarcinoma of the pancreas. Arch Surg 127: 1335-1339.

17. Ueno H, loka T, Ikeda M, Ohkawa S, Yanagimoto H, et al. (2013) Randomized phase III study of gemcitabine plus S-1, S-1 alone, or gemcitabine alone in patients with locally advanced and metastatic pancreatic cancer in Japan and Taiwan: GEST study. J Clin Oncol 31: 1640-1648. 
Citation: Matsushita A, Nakamura Y, Sumiyoshi H, Aimoto T, Yokoyama T, et al. (2013) A Pilot Study- Neoadjuvant Chemotherapy with Gemcitabine and S1 in Patients with Resectable and Borderline Resectable Pancreatic Cancer. J Carcinogene Mutagene S9: 006. doi:10.4172/21572518.S9-006

Page 5 of 5

18. Varadhachary GR, Wolff RA, Crane CH, Sun CC, Lee JE, et al. (2008) Preoperative gemcitabine and cisplatin followed by gemcitabine-based chemoradiation for resectable adenocarcinoma of the pancreatic head. $\mathrm{J}$ Clin Oncol 26: 3487-3495

19. Turrini O, Viret F, Moureau-Zabotto L, Guiramand J, Moutardier V, et al. (2009) Neoadjuvant 5 fluorouracil-cisplatin chemoradiation effect on survival in patients with resectable pancreatic head adenocarcinoma: a ten-year single institution experience. Oncology 76: 413-419.

20. Patel M, Hoffe S, Malafa M, Hodul P, Klapman J, et al. (2011) Neoadjuvant GTX chemotherapy and IMRT-based chemoradiation for borderline resectable pancreatic cancer. J Surg Oncol 104: 155-161.

21. Sahora K, Kuehrer I, Eisenhut A, Akan B, Koellblinger C, et al. (2011)
NeoGemOx: Gemcitabine and oxaliplatin as neoadjuvant treatment for locally advanced, nonmetastasized pancreatic cancer. Surgery 149: 311-320.

22. Lee JL, Kim SC, Kim JH, Lee SS, Kim TW, et al. (2012) Prospective efficacy and safety study of neoadjuvant gemcitabine with capecitabine combination chemotherapy for borderline-resectable or unresectable locally advanced pancreatic adenocarcinoma. Surgery 152: 851-862.

23. Hosein PJ, Macintyre J, Kawamura C, Maldonado JC, Ernani V, et al. (2012) A retrospective study of neoadjuvant FOLFIRINOX in unresectable or borderlineresectable locally advanced pancreatic adenocarcinoma. BMC Cancer 12: 199.

24. Conroy T, Desseigne F, Ychou M, Bouché O, Guimbaud R, et al. (2011) FOLFIRINOX versus gemcitabine for metastatic pancreatic cancer. N Engl Med 364: 1817-1825. 